\title{
A HYBRIDIZATION OF ARTIFICIAL NEURAL NETWORK AND SUPPORT VECTOR MACHINE FOR PREVENTION OF ROAD ACCIDENTS IN VANET
}

\section{Chiranjit Dutta}

Research Scholar, Department of Computer Science and Engineering, Shobhit Institute of Engineering and Technology, Uttar Pradesh, India

\section{Dr. Niraj Singhal}

Professor, Department of Computer Science and Engineering, Shobhit Institute of Engineering and Technology, Uttar Pradesh, India

\begin{abstract}
Vehicular Ad hoc Network (VANET) is known as an infrastructure less network having dynamic nodes with Road Side Units (RSUs). Data Broadcasting becomes a very difficult task because of more density, scalability, randomness, mobility of vehicles. VANET has an ability to prevent accidents by transmitting data on-time on the network and this has raised an attention for number of researchers. Therefore, in this paper a realistic mechanism has been proposed to avoid the fatal accidents on road using clustering approach with the concept of Artificial Intelligence. Hybridization of Artificial Neural Network (ANN) and Support Vector Machine (SVM) is conducted to speed up the data transmission process that assists in providing information accurately and on-time. To demonstrate the efficacy of the novel mechanism, parameters such as Throughput, Packet Delivery ratio (PDR) are considered.
\end{abstract}

Keywords: ANN, RSUs, SVM, VANET

Cite this Article: Chiranjit Dutta and Dr. Niraj Singhal, a Hybridization of Artificial Neural Network and Support Vector Machine for Prevention of Road Accidents in Vanet, International Journal of Computer Engineering and Technology, 10(01), 2019, pp. (110)-(116).

http://www.iaeme.com/IJCET/issues.asp?JTypeIJCET\&VType=10\&IType=1

\section{INTRODUCTION}

Over the past few decades, the number of vehicles has significantly increased which has resulted in the populated roads. This massive traffic load has uplifted the road accident graph, which has consequently raised injuries and death rates worldwide [1]. According to data from the Highway Transport and Highways Department, the number of road traffic fatalities 
increased by $31 \%$ from 2007 to 2017 . Since 2005 , the number of fatal accidents has increased steadily from 1, 31,726 in 2015 to 1 person and 36,071 in 2016 [2]. Due to the lack of security measures, India faces a high incidence of accidents in the world. Therefore, the focus is on improving road safety measures to avoid vehicle collisions and road accidents. Varied safety measures to avoid road accidents like traffic monitoring and channelling works with technology such as alarm systems and information dissemination [3]. Vehicular Ad hoc network (VANET) is a technology that is now widely utilized for the prevention of road traffic accidents. It is a network that communicates between vehicles and infrastructure. It is often referred to as the Network on Wheels. For the safety of passengers, warning messages are shared between vehicles [4]. Warning messages concerned with traffic signal violations, road conditions, and traffic congestion and so on. VANET communication is categorized in two ways, namely Vehicle-To-Vehicle Communication (V2V), Vehicle-To-Infrastructure (V2I) [5]. In V2V communication, limited communication services such as Wi-Fi and Wireless Access in Vehicular Environment (WAVE) are utilized. Numerous efforts have been made to avoid such drastic situations through the implications of speed limits, imposition of traffic rules, deployment of seat belts and air bags, boosting stiffness into the physical structure of a vehicle and so on [6]. But till data, the expected reduction in road accidents has not been achieved. So, in this research an effort has been made to shun away the road accidents by utilizing an approach of clustering with Road Side Units monitoring using Artificial Intelligence.

\section{RELATED WORK}

Lozano et al. [1] has proposed a warning information scheme to avoid traffic accidents between vehicles and to issue a warning message to remind driver of the current accident situation. The proposed solution uses a distance-based flooding scheme. Gokulakrishnan et al. [2] discovered an accident avoidance routing scheme called Road Accident Prevention (RAP). The program introduced an Early Warning (EW) message to make the necessary decisions - choose an alternate route, slow down the vehicle and change lanes. Dawood et al. [3] proposed an accident avoidance scheme called Efficient Emergency Message Broadcasting (EEMB) routing scheme. The goal of research is to lessen traffic bottlenecks and shun away different traffic accidents by broadcasting emergency information at high rates and low overhead.

Benslimane et al. [4] has believed that the user having emergency message should broadcast the message constantly till it chooses other forwarder vehicle. However, since multiple broadcast attempts cannot help the vehicle to acquire another vehicle, this propagation results in a repetition of the broadcast message. Therefore, the traffic network will be affected by the processing overhead. To minimize processing overhead, the authors proposed a solution called Optimized Dissemination of Alarm Message (ODAM) that also limited to regional and freight vehicles. Roy et al. [5] presented a mechanism to avoid accidents and prevent congestion in the vehicle environment. In this scenario, the affected vehicle generates an emergency message after the accident. Road Side Unit receives the emergency message and then resends another emergency message to other Road Side Units located within its range. Kumar et al. [6] presented a distance-based routing mechanism. The objective of this research is to avoid accidents at intersections because most of the time anonymous vehicles appears from the other side of the road and suffers from accident.

Kshirsagar et al. [7, 8] presented an intelligent vehicle management solution to avoid traffic accidents. The proposed solution uses basic warning safety information to avoid traffic congestion. Traffic signals and management solutions have successfully achieved high throughput, high delivery rates and low latency. Nzouonta et al. [9] proposed a method termed as Spatio-Temporal Emergency Information Dissemination (STEID). The objective of this 
routing scheme is to propose hybrid network architecture by using cellular links and proxy servers.

\section{CLUSTERING BASED VANET ARCHITECTURE}

In non-urban regions, there are inadequate roadside connections for data communication. Every vehicle for effective vehicle communication acts as a router for data exchange. Broadcasting and routing algorithms utilizes Global Positioning System (GPS) data to communicate efficiently. For road security, in this research novel applications for vehicle networks, i.e., travel and tourism information dispersion, car to car communication with game applications. Clustering based Vehicular Architecture is shown below in fig 1.

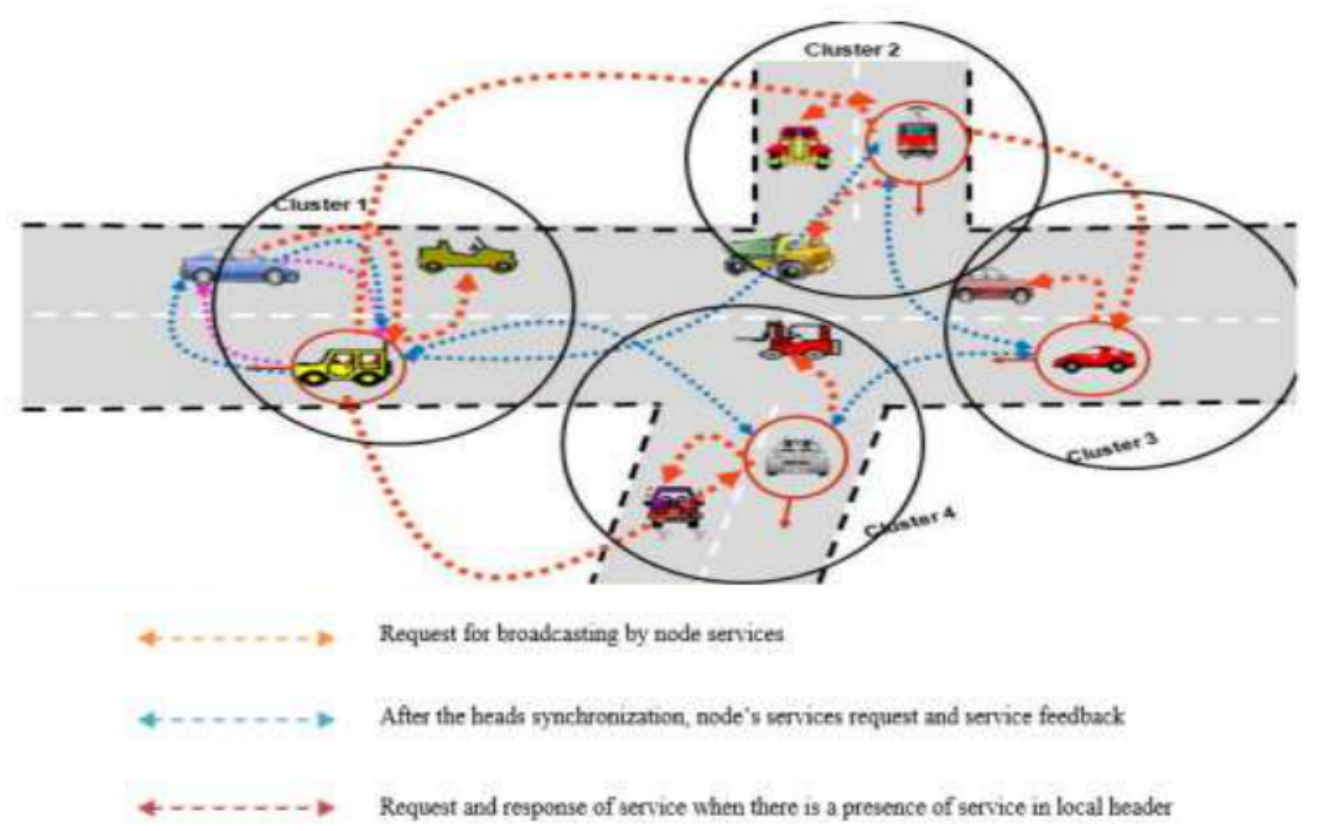

Figure 1 Vehicular Architecture with Clusters

These applications require a consistent connection among high data rates and a stable connection among transmitter and receiver in high reliability situation. In the proposed model, the Cluster creation procedure varies from the traditional model. The size of cluster differs inevitably as a result of an abrupt increase in the number of vehicles moving on a certain path just because of traffic fluctuations. The cluster stays at the same speed, so the cluster areas are created as constant. Creating a cluster should guarantee that Cluster Head $(\mathrm{CH})$ does not exceed the cluster border frequently. If the vehicles move regularly, the cluster head selection algorithm regularly selects a novel cluster. VANET area is divided into several groups using the proposed cluster formation algorithm. Every cluster has a Cluster Head and the $\mathrm{CH}$ can either be a Road Side Unit or a good database of any of the storage and retrieval facilities. Every cluster head has all the service tariffs available on the network. VANET's entire CHs are frequently updated, even if they are novel service center on the network.

\section{PROPOSED ALGORITHM}

The proposed algorithm is designed using hybridization of Support Vector Machine along with Artificial Neural Network to improve data transmission rate using the vehicles and RSU properties being categorized by clustering technique. 
A Hybridization of Artificial Neural Network and Support Vector Machine for Prevention of Road Accidents in Vanet

\section{Algorithm: Support Vector Machine based Artificial Neural Network}

Input: Properties of Vehicular and RSU (T) using Clustering, Types of Vehicles (Cat), Kernel Function and Neurons (N)

Output: Authorized Vehicles

\section{Training:}

1 Initialize the SVM training data, T is the total clustered vehicles and RSU property with Radial Basis Function (RBF) as Kernel function

2 for $\mathrm{I}=1 \rightarrow$ All Vehicles

3 if Property of Node (I) == Real

4 Defined the Cat as a categories of training data

5 Cat $(1)=$ Vehicles $(I)$

6 else

7 Cat (2) $=$ Vehicles (I)

8 end

9 end

10 Train_Structure=SVMTRAIN (T, Cat, Kernel function)

$11 \mathrm{~T}=$ Train_Structure.SupportVector //To find out the training data for ANN

12 Initialize the basic parameters of ANN

- Number of Epochs (E) // Iterations used by ANN

- Number of Neurons ( $\mathrm{N}$ )

- Performance: MSE, Gradient, Mutation and Validation

-Techniques: Levenberg Marquardt

- Data Division: Random

13 for $\mathrm{i}=1 \rightarrow \mathrm{T}$

14 if $\mathrm{T}$ belongs to real vehicle cluster property

15 Group (1) = Properties of training data according to the real vehicles

16 else if $\mathrm{T}$ belongs to non-real vehicle cluster property

17 Group (2) = Properties of training data according to the non-real vehicles

18 else

19 Group (3) = Extra properties of training data

20 end

21 end

22 Initialized the ANN using Training data and Group

$23 \mathrm{Net}=\operatorname{Newff}(T$, Group,$N)$

24 Set the training parameters according to the requirements and train the system

$25 \mathrm{Net}=$ Train (Net, Training data, Group)

\section{Testing:}

26 Current Vehicles $=$ Properties of current vehicle in network

27 Authorization $=$ simulate $($ Net, Current Vehicles)

28 if Authorization is valid

29 Authorized Vehicles $=$ Right 
30 else

31 Authorized Vehicles $=$ Wrong

32 end

33 Return; Authorized Vehicles as output

34 end

In this algorithm, firstly we have used Support Vector Machine (SVM) using the Radial Basis Function (RBF) as a kernel and most helpful properties are discovered for ANN known as support vectors. Support vectors are best data of network which is selected by the RBF kernel function. After that the ANN is used to train and classify the vehicles authority which helps to improve the performance of network. If the selection of network is better, then the speed of simulation is faster which is shown in the next segment of this paper.

\section{RESULT AND DISCUSSION}

To depict the capability of novel mechanism, execution of varied parameters such as Packet Delivery Ratio (PDR), Throughput is conducted. The results obtained after the computation are demonstrated below in tabular and graphical form:

Table I Computation of Packet Delivery Ratio

\begin{tabular}{|c|c|c|}
\hline No. of Rounds & PDR With ANN & PDR With SVM+ANN \\
\hline 20 & 0.631 & 0.674 \\
\hline 50 & 0.615 & 0.683 \\
\hline 100 & 0.638 & 0.693 \\
\hline 150 & 0.639 & 0.715 \\
\hline 200 & 0.655 & 0.722 \\
\hline 250 & 0.668 & 0.749 \\
\hline 300 & 0.671 & 0.732 \\
\hline 500 & 0.672 & 0.753 \\
\hline
\end{tabular}

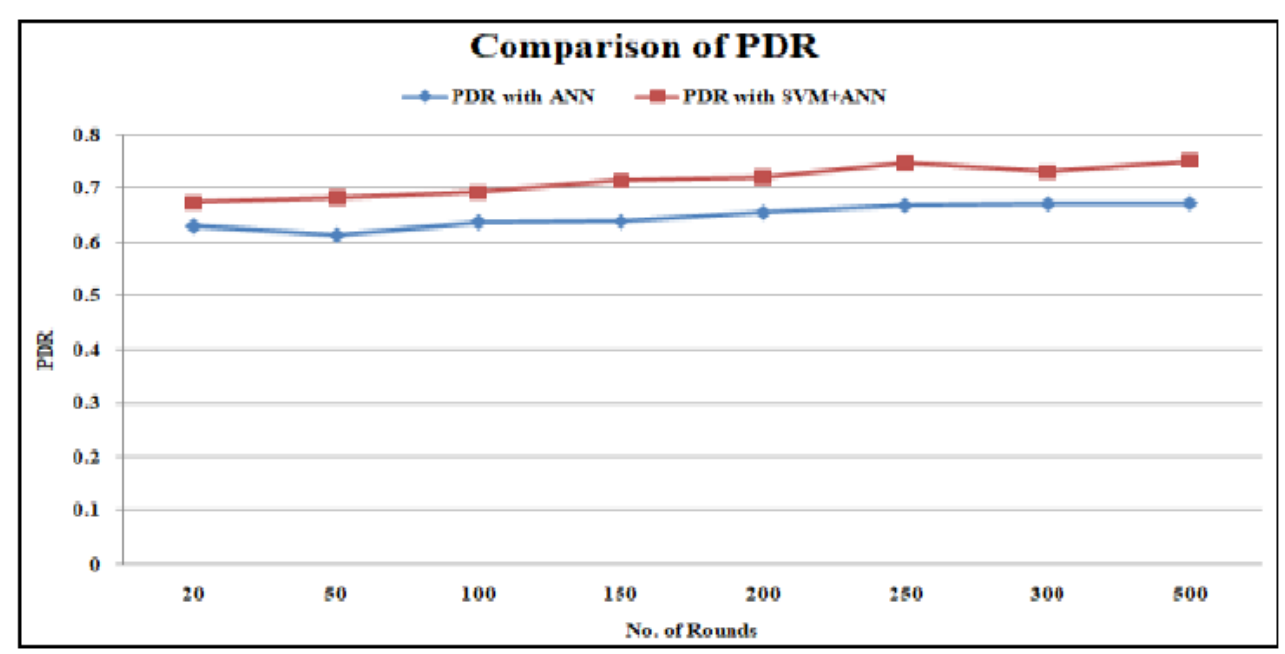

Figure 2 Packet Delivery Ratio Comparison

Table I and fig 2 shows the computation and comparison of Packet Delivery Ratio (PDR). The computation has been drawn by contrasting ANN and hybridization of SVM and ANN comparison. PDR helps to detect the speed by which the data has been transferred from vehicle to vehicle and from vehicle to RSUs. Number of rounds taken for the execution is 500 shown in X-axis of the figure and Y-axis shows the PDR obtained values. The average value of PDR 
A Hybridization of Artificial Neural Network and Support Vector Machine for Prevention of Road Accidents in Vanet

with ANN is 0.648 and the average value of PDR with ANN and SVM is 0.715 . The computation and comparison of throughput with ANN and with SVM+ANN is shown below in Table II and fig 3.

Table II Computation of Throughput

\begin{tabular}{|c|c|c|}
\hline No. of Rounds & Throughput With ANN & Throughput With SVM+ANN \\
\hline 20 & 88.31 & 92.94 \\
\hline 50 & 89.29 & 94.73 \\
\hline 100 & 87.33 & 92.23 \\
\hline 150 & 91.83 & 95.38 \\
\hline 200 & 89.38 & 97.48 \\
\hline 250 & 90.23 & 93.85 \\
\hline 300 & 88.38 & 93.36 \\
\hline 500 & 93.53 & 96.51 \\
\hline
\end{tabular}

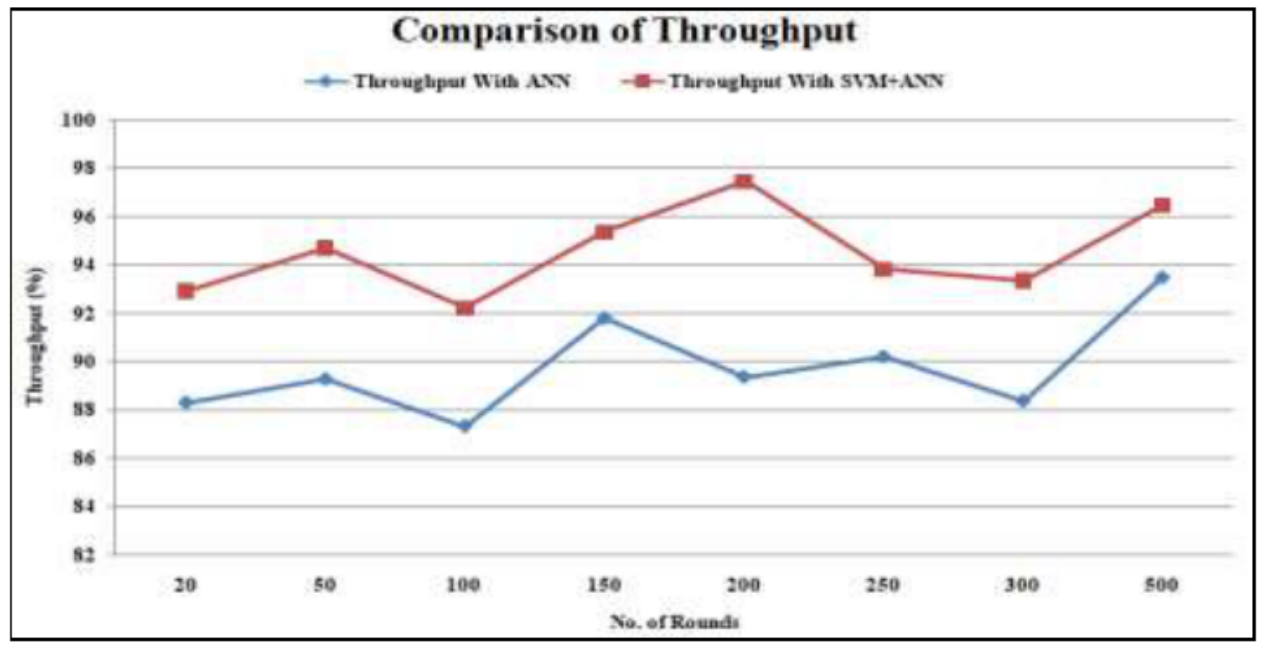

Figure 3 Throughput Comparison

For the comparison, 500 rounds are taken. Throughput helps in considering utmost data while data broadcasting process is done between vehicle to vehicle and from vehicle to RSU. The average value of throughput in case of ANN is 89.78 whereas average value of throughput in case of ANN and SVM is 94.56. It can be seen that the proposed hybridization mechanism has more throughput as comparative to ANN only.

\section{CONCLUSION}

As the Indian population is second largest in the world, so vehicles are increasing per day and simultaneously accidents are rising. There is no security for human life and the vehicles. VANET is the mostly utilized network that communicates among the vehicles. It is not only utilized for the communication purpose but also could be structured for navigation and traffic control. In this manuscript, a mechanism has been lightened that would help in the reduction of accidents. Hybridization of Artificial Intelligence (AI) techniques is done that helps in accelerating data transmission and authentication. Varied QoS parameters are computed to depict the effectiveness of the proposed work. The average value of PDR with ANN is 0.648 and the average value of PDR with ANN and SVM is 0.715. The average value of throughput in case of ANN is 89.78 whereas average value of throughput in case of ANN and SVM is 94.56 . 


\section{REFERENCES}

[1] Garcia Lozano, E. Barba, C.T.Igartua and M.A. Campo C., A distributed, bandwidthefficient Accident Prevention System for interurban VANETs, Proceedings of the International Conference on Smart Communications in Network Technologies ( $\mathrm{SaCoNeT}$ ), Paris, France, pp. 17-19, June 2013.

[2] Gokulakrishnan P. and Ganeshkumar P, Road Accident Prevention with Instant Emergency Warning Message Dissemination in Vehicular Ad-Hoc Network, PLoS ONE, 10, e0143383, 2015.

[3] Dawood H.S. and Wang Y., An Efficient Emergency Message Broadcasting Scheme in Vehicular Ad Hoc Networks, International Journal of Distributed Sensor Networks, 11, 232916 [CrossRef], 2013.

[4] Benslimane A., Optimized Dissemination of Alarm Messages in Vehicular Ad-Hoc Networks (VANET), Proceedings of the 7th IEEE International Conference on High Speed Networks and Multimedia Communications, Toulouse, France, pp. 655-666, 30 June-2 July 2004.

[5] Roy A. and Chakraborty J., Communication based Accident Avoidance and Congestion Control mechanism in VANETs, Proceedings of the International Symposium on Advanced Computing and Communication (ISACC), Silchar, India, pp. 320-327, 14-15 September 2015.

[6] Kumar R.R., Begum S.W. and Manikandan M., Distance Based Accident Prevention in Intersection using VANET, International Journal of Innovative Research in Computer Communication Engineering, pp. 3624-3629, 2007.

[7] Kshirsagar N.S. and Sutar U.S., Review on Intelligent Traffic Management System Based on VANET, International Journal of Innovative Research in Computer Communication Engineering, 3, pp. 2001-2004, 2015.

[8] Kshirsagar, N.S. and Sutar U.S., An Intelligent Traffic Management and Accident Prevention System based on VANET. International Journal and Science Research, 2013.

[9] Nzouonta J. and Borcea C., A Protocol for Emergency Information Dissemination in Vehicular Networks, Report; Department of Computer Science, New Jersey Institute of Technology: Newark, NJ, USA, 2006.

[10] Goniewicz K., Goniewicz M., Pawłowski W. and Fiedor P, Road Accident rates: Strategies and programmes for improving road traffic safety, European journal of trauma and emergency surger, 2016.

[11] Soehodho S., Public Transportation Development and Traffic Accident Prevention in Indonesia, International Association of Traffic and Safety Sciences Research, 40(2), pp.7680, 2017.

[12] Kumar S. and Toshniwal D., Analysing Road Accident data using Association rule mining, International Conference on Computing, Communication and Security (ICCCS), IEEE, pp. $1-6,2015$.

[13] Entezami N. et al., Epidemiology of fatal road traffic accidents in Northern provinces of Iran during 2009 to 2010, Safety Promotion and Injury Prevention, 3(1), pp. 1-8, 2015.

[14] Al Najada H. and Mahgoub I, Big vehicular traffic data mining: Towards Accident and Congestion prevention, International Wireless Communications and Mobile Computing Conference (IWCMC), IEEE, pp. 256-261, 2016.

[15] Etehad H. Et al., Impact of Road Traffic Accidents on the elderly, Archives of gerontology and geriatrics, 61(3), 489-493, 2015.

[16] Sharma S. M., Road Traffic Accidents in India, International Journal of Advanced and Integrated Medical Sciences, 1(2), 57-64, 2016. 